\title{
CDISC SDTM Cardiac Rhythm Device Failure Manifestation Terminology
}

National Cancer Institute

\section{Source}

National Cancer Institute. CDISC SDTM Cardiac Rhythm Device Failure Manifestation

Terminology. NCl Thesaurus. Code C101866.

Terminology associated with the cardiac rhythm device failure manifestation codelist of the Clinical Data Interchange Standards Consortium (CDISC) Study Data Tabulation Model (SDT M). 